\title{
FACE PROFILE RECOGNITION AND IDENTIFICATION
}

\author{
Eng. Shimaa M.Hani*, Dr. Hesham Keshk*, Dr. Tarek El- \\ Tobely** and Prof. Dr. Mohamed El-Adawy* \\ * Electronics, Communications \& Computer Department, Helwan \\ University, Egypt \\ **Computer and Automatic Control Department, Tanta University, Egypt \\ email:shimahani82more@hotmail.com
}

(Received January 27, 2011 Accepted April 2, 2011)

Since the early 1990's face Recognition Technology (FRT) became an active research area. Most of the current profile recognition algorithms depend on the correct detection of fiducial points and the determination of relationships among these fiducial points. Unfortunately, some features such as concave nose, lips, flat chin, etc. make detection of such points difficult and unreliable. Also the number and position of fiducial points vary when pose changes even for the same person. In this paper, a curvature-proposed technique is presented, which does not require the extraction of all the fiducial points, but uses information contained in the profile. The nearest neighbor interpolation method is used to smooth the profile and then the curvature of the interpolated profile is computed. Using the curvature coefficient values, the fiducial points, such as nasion, chin, and forehead can be reliably extracted using a fast and simple method. Then an Euclidean distance method is applied to match the face profile based on the curvature coefficient values.

Experiments are performed on collected 50 clients with different ages, in public area. Each had three shots differences in time capture and illuminations, getting total of 150 images database, recognition rate of $96.67 \%$ and conclusion are presented and discussed.

KEYWORDS - face profile; curve fitting; face recognition; human features, curvature; facial extraction, facial motion; facial expression; nasal detection and identification.

\section{INTRODUCTION}

Face profile is an important aspect for the recognition of faces, which provides a complementary structure of the face that is not seen in the frontal view. Though it inherently contains less discriminating power than frontal images, it is relatively easy to analyze and more foolproof. Within the last decade, several algorithms have been proposed for automatic person identification using face profile images. The commonly used methods for face profile recognition include: Scale space filtering [1], Morphological transform [2, 3], Dynamic time warping [4], Attributed string [5], and Hidden Markov model [6, 7].

In the framework of profile analysis, early work by Zadravko, and Loncaric [1] used Gaussian convolution and had twenty one fiducial points of ten distances (D1D10) and eleven profile arcs (A1-A11), and they used three feature vectors description 
using morphological operations (dilation \& erosion) for hair growth and hair cut [2]. Later, Yongsheng and Maylor used a new attributed string matching for human face profile recognition by merging dominant string matching $(\mathrm{m}$ and $\mathrm{n})$ are the lines, segment numbers of strings (A and B) [5]. Maja \& Ioannis and Leon used motion estimation and color skin initialization and define feature parameters by encode facial action units (AU) [8]. Curvature estimation based fiducial extraction by Xiaoli and Bir Bhanu [4] and more recently worked by extraction of face profile images from video by a correlation based method using threshold to get high resolution side face image reconstruction from Low-resolution side face images, used dynamic time warping (DTW) matching method for face profile recognition [4].

Recently, Maja Pantic \& Ioannis Patras used 15 facial points introduce facial (action unit: AU). He took dynamic recognition from continuous video input, and expression pictures [9].

Frank Wallhoff, and Gerhard Rigoll [7] in 2001, presented an approach to recognize profile views (90 degree) with a system trained on transformed frontal views. The system combined an Artificial Neural Network (ANN) and a classification process based on Hidden Markov Models (HMM). The presented system has been tested with subsets of the FERET and the MUGSHOT databases; and a maximum recognition score of $42 \%$ was measured.

In this work, we try to develop a simple and fast method for detecting the fiducial points. For that purpose, we treat the outline of a profile as a function and we use curve fitting algorithm for detection of convex and concave in that line of the profile by its first and second derivatives. From this curvature image, we determine parameters which are used for detection of specific fiducial points. A set of twentyeight feature characteristics is derived from these fiducial marks. After normalizing the feature characteristics using two selected fiducial marks, Euclidean distance measure was used for measuring the similarity of the feature vectors derived from the outline profiles. Results of the proposed profile matching method in the presence of profile faces are included in this paper.

\section{FACE RECOGNITION APPROACHES}

Biometric recognition is a digital analysis using cameras or scanners of biological characteristics such as facial structure, fingerprints and iris patterns to match profiles to databases of people. Face recognition is the ability of a computer to scan, store, and recognize human faces for use in identifying people. There are three approaches of face recognition:

\subsection{Face Frontal Recognition:}

In the face recognition the features are used through the frontal side of eyes, nose, mouth, checks, eyebrows and sometimes ears. Face frontal recognition is of great importance in many applications such as personal identification, employee access to high security areas. Face frontal recognition approaches are adversely affected by varying lighting conditions and particularly with respect to varying pose. Face represented in a frontal view with wide expression change and has failed to match up to expectations: Variations in pose, illumination and expression limit the performance of frontal recognition techniques [10]. 
Rein-Lien, Mohamed Abdel-Mottaleb and Anil K. Jain [11] used face detection algorithm for color images in the presence of varying lighting conditions with a complex backgrounds. Based on a novel lighting compensation technique and nonlinear color transformation. They detected skin regions over the entire image and then generated face candidates. There algorithm constructed eye, Mouth, and face boundary to verify the face candidates. They used two databases HHI and Champion databases. There head pose were Frontal, Near-Frontal, Half-Profile, and Profile.

Recently, Yin Zhang, Zhi-Hua [12] formulated the face recognition problem as a multi-class cost-sensitive, and developed two theoretically methods: 1) mcKLR: multi-class Kernal logistic regression, it is an inductive learning method derived from Bayes decision theory. 2) mckNN: multi-class k-nearest neighbor. They used Four different kinds of features were extracted from the face images by using: Principle Component Analysis (PCA), Locality Preserving Projections (LPPs), Linear Discriminate Analysis (LDA) and Local Binary Pattern (LBP). They used two databases AR and FERET databases. They proved that the performance of mcKLR is always the best except with LDA where the mckNN was a good choice.

\subsection{Face Profile Recognition:}

Face profile is minimally affected by different expressions and the nose is not changed at all. In the same way, the face profile is hard to be changed unless a radical change is performed, as if a plastic surgery is done or accident occurred involving the facial bone. For these reasons, the face profile is providing a great benefit to identify criminals. Therefore, the present study indicated that the right hemisphere was dominant for the perception of profile views as well as frontal views. Human face processing studies on different view faces suggested that the frontal and the profile views contain different information of the face. The frontal faces convey the internal feature information; such as eyes, nose, and mouth, on the contrary, the profile view face convey three-dimensional shape information; such as the nose height and shape of the chin. These two types of information are independent, and each of these is important for face processing, and this work is about to explain the role of the nose in face profile recognition.

Xiaona $\mathrm{Xu}$, Zhichun $\mathrm{Mu}$ [13] used a novel non-intrusive multimodal recognition technology based on ear and profile face. The face profile-view captured for recognition, then ear classifier and profile face classifier based on Full-Space Linear Discriminant Analysis (FSLDA) were set up. They used decision fusion of ear and profile face, carried out using the combination methods of Product, Sum, and Median rules according to the Bayesian theory and a modified Vote rule the two classifiers.

I.A. Kakadiaris, H. Abdelmunim, W. Yang, and T. Theoharis [14] introduced a new system for profile-based face recognition. There scenario involved a driver entering a gated area and using his/her side-view image (the driver remains seated in the vehicle) as identification. The system have two modes: enrollment and identification. In the enrollment mode, 3D face models of subjects were acquired and profile extracted under different poses and stored to form a gallery database. In the identification mode, 2D images were acquired and the corresponding planer profiles were extracted and used as probes. Then, probes were matched to the gallery profiles to determine identity. 


\subsection{Three Dimensions Face Recognition:}

The first step, toward the development of a 3D face recognition solution for the intensity image domain based on 2D face recognition (frontal, profile). There are a number of questions 3D face recognition research needs to address. In acquisition, the accuracy of cheaper and less intrusive systems needs to be improved, temporal sequences should be considered [10]. This approach is expensive to implement and need more computations.

Mohammed H. Mahoor, A-Nasser and Mohamed Abdel-Mottaleb [15] unified a graph model, called Attributed Relational Graph (ARG), for multi-modal face modeling and recognition. It is based on the ARG modal, the $2 \mathrm{D}$ and $3 \mathrm{D}$ data were included in a single model. They developed ARG model consists of nodes, edges, and mutual relations. The nodes of the graph corresponded to the landmark points were extracted by an improved Active Shape Model (ASM) technique (at each node of the graph, they responded to a set of log-Gabor filters to the facial image texture then shape information (depth values) was calculated). The edges of the graph were defined based on Delaunay triangulation and a set of mutual relations between the sides of the triangles were defined. They fused 2D and 3D attributes and the mutual relations at the score level by the University of Miami face datadase.

Ansari A-Nasser, Mahoor Mohammed, and Abdel-Mottaleb Mohamed [16] illustrated a modal-based approach that represented the 3D facial data of a given subject by a deformed 3D mesh model for face recognition. The system consisted of two stages: 1) modeling stage, only three facial feature points were extracted from the range image and then used to aligned the $3 \mathrm{D}$ generic face model to the entire range of a given subject's face. Then each aligned triangle of the mesh model, with three vertices, was treated as a surface plane which was fitted (deformed) to its corresponding interior 3D range data, used least square plane fitting. Via triangular vertices subdivisions, a higher resolution model was generated from the coordinates of the aligned and fitted model. Finally, the model and its triangular surfaces were fitted once again resulting in a smoother mesh model that resembled and captured the surface characteristic of the face. In the recognition stage, a 3D probe face was similarly modeled and compared to all faces in the database.

Steven Cadavid, Mohammed H. Mahoor, and Mohamed Abdel- Mottaleb [17] used a multi- modal ear and face biometric system. The system was comprised of two components: a 3D ear recognition component and a $2 \mathrm{D}$ face recognition component. For the 3D ear recognition, a series of frames was extracted from a video clip and the region of interest (i.e., ear) in each frame was independently reconstructed in 3D using Shape From Shading (SFS). The resulting 3D models were then registered using the iterative closest point (ICP) algorithm. For the $2 \mathrm{D}$ face recognition, a set of facial landmarks was extracted from frontal facial images using the Active Shape Model (ASM). The Gabor features (attributes) were stored in the database as the face model for recognition. The match scores of the ear recognition and face recognition modalities were fused to boost the overall recognition rate of the system and achieved using the weighted sum technique for fusion.

Mohammed H. Mahoor, Mohamed Abdel- Mottaleb [18] used the principal curvature to represent the face image as a $3 \mathrm{D}$ binary image called ridge image. The ridge image showed the locations of the ridge points around the important facial 
regions on the face (i.e., the eyes, the nose, and the mouth). The robust were utilized for Hausdorff distance and the iterative closest points (ICP) for matching the ridge image of a given probe image to the ridge images of the facial images in the gallery. They performed experiments on GavabDB face database (a small size database) and Face Recognition Grand Challenge (a large size database). The results showed that the ridge lines had great capability for 3D face recognition.

Chaua C. Queirolo, Luciano Silva, Olga R.P. Bellon, and Mauricio Pamplona Segundo [19] used a Simulated Annealing-based approach (SA) for range image registration with the Surface Interpenetration Measure (SIM), as similarity measure, in order to match two face images. The authentication score was obtained by combining the SIM values corresponded to the matching of four different face regions: circular and elliptical areas around the nose, forehead, and the entire face region. Then, a modified SA approach was proposed taking advantage of invariant face regions to better handle facial expressions. They performed on the FRGC v2 database, the largest available database of 3D face images composed of 4,007 images with different facial expressions.

Ira Kemelmacher-Shlizerman, Ronen Basri [20] used a mere single 3D reference model of a different person's face. Classical reconstruction methods from single images, i.e., shape from-shading (SFS), required knowledge of the reflectance properties and lighting as well as depth values for boundary conditions. They represented the input faces as combinations (of hundreds) of stored 3D models. They used the input image as a guide to "mold" a single reference model to reach a reconstruction of the sought 3D shape. They used Lambertian reflectance and harmonic representations of lighting.

\section{PROPOSED TECHNIQUE}

In common face profile approach, features are extracted from the hole profile and this sometimes miss leads the recognition process due to the variations in the profile of the same person used different facial expressions. However, the nose area in the face profile is not affected by the facial expressions profile. For this reason, we extract the nose only and then extract some features of the nose and these features will be used for classification. Among many methods that are available for classification we used the Euclidean distance as the matching method due to its robustness and simplicity. The overall steps of the proposed technique is shown in figure (1).

\subsection{Preprocessing}

In this stage, the RGB image is converted into a binary image, then the edges are determined and the face outer profile (face contour) is detected as shown in figure(2). The RGB image shown in figure (2-a), binary image shown in figure (2-b), edges image shown in figure (2-c), and the outer profile shown in figure (2-d). The noisy pixels are filtered by window $5 * 5$ and the missing pixels in the face contour are estimated by the nearest neighbor algorithm [21]. 


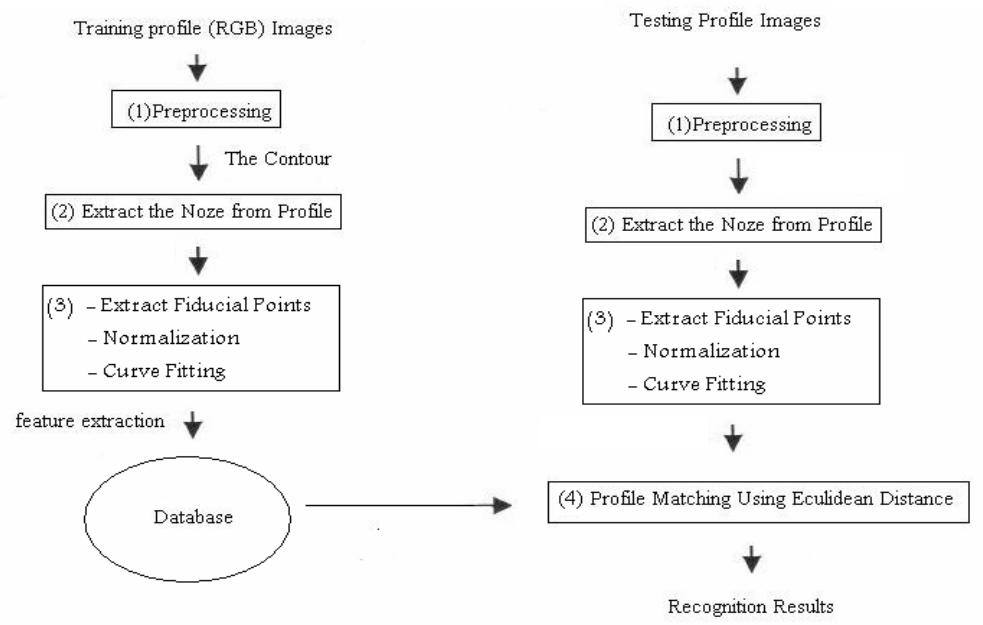

Figure (1) The Frame of the face Profile Recognition Approach

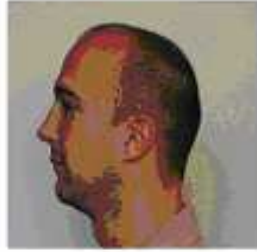

(a)

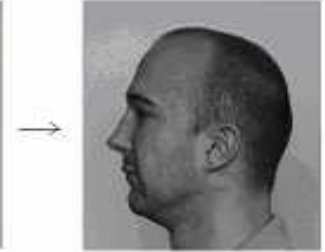

(b)

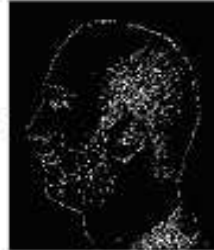

(c)

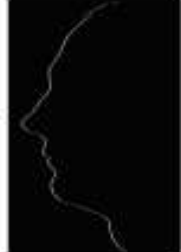

(d)

Figure (2) Detection of the face outer profile

\section{2. land mark detection}

In this work, only the nasion area in the face profile is used in the feature extraction. Three fiducial land mark points in the nasion area are used to find out the pixels of this area. These three land mark points are selected as follow:

Point 1 (tip): The left most point in the middle of the face profile.

Point 2 (top): The first point with maximum curvature above tip point.

Point 3(bottom): The first point with maximum curvature below tip point.

The selected tip, top, and bottom points are shown in figure (3).

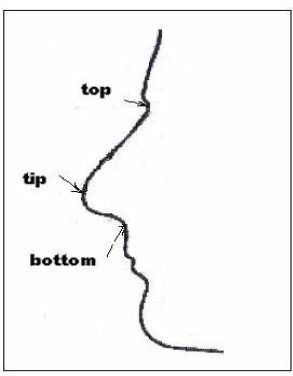

Figure (3) nose main points 


\subsection{Curve Fitting}

Curve fitting is the process of constructing a curve that has the best fit to a series of data points. In our approach, curve fitting technique is used to eliminate high frequency noise in the face profile. The constructed curve starts at the top land mark point and ends at the bottom land mark point. Figure(4) shows the constructed curve for nose pixels. The curve fitting process is implemented by the following polynomial, where $a_{n}$ is a constant coefficient. These polynomial coefficients will be considered as the features introduced to the classifier. The general form for a linear constant coefficient is as follows:

$\mathrm{y}=\mathrm{a}_{\mathrm{n}} \mathrm{x}^{\mathrm{n}}+\mathrm{a}_{\mathrm{n}-1} \mathrm{x}^{\mathrm{n}-1}+\mathrm{a}_{\mathrm{n}-2} \mathrm{x}^{\mathrm{n}-2}+\ldots . .+\mathrm{a}_{0} \mathrm{x}^{0}$

Where, $\mathrm{n}$ : is the order of the polynomial. In this paper, the $10^{\text {th }}$ order is chosen by the trial and error to appropriate to the images in the database and to be accepted for time consuming.

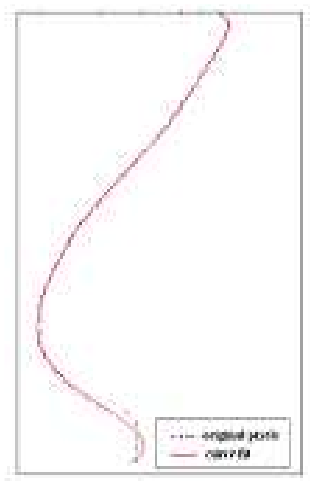

Figure (4) nose with $10^{\text {th }}$ order polynomial curve fitting

\subsection{Fiducial extraction}

Until now, the pixels of the nasion area starting at top land mark point and ending at bottom land mark point are extracted, smoothed, and normalized by taking the maximum distance and dividing all distances over its value, and abstracting the slope between the contour and the vertical axis for each image. In this proposed the features of the nose area will be extracted as follows:

- A line connecting the top land mark point and the bottom land mark point is drown.

- This line is divided into $\mathrm{m}$ local points at equal distance.

- The distances between nose contour and the local points are measured: $\left(\mathrm{d}_{1}-\mathrm{d}_{\mathrm{m}}\right)$.

- The slope of each nose contour segment is measured with reference to the line connecting the top land mark point and the bottom land mark point: $\left(\alpha_{1}-\alpha_{m}\right)$.

Finally $d_{1}-d_{m}$ and $\alpha_{1}-\alpha_{m}$ are used as the fiducial features vector for each face profile as shown in figure (5-a) and figure (5-b).

\subsection{Profile matching}

The Euclidean distance is used to measure the similarities between the feature vectors of any two face profiles. If $\mathrm{k}$ is dimension of the feature vector, then $\mathrm{U}_{\mathrm{i}}$ and $\mathrm{V}_{\mathrm{i}}$ are the 
facial features of profile $\mathrm{U}$ and $\mathrm{V}$, respectively, ED is the distance between the feature vector $U$ and $V$. This is measured for both the distance features $\left(d_{1}-d_{m}\right)$ and the slope features $\left(\alpha_{1}-\alpha_{m}\right)$.

$E D=\min \left(\sqrt{\sum_{i=1}^{h}\left(U_{i}-V_{i}\right)^{2}}\right)$

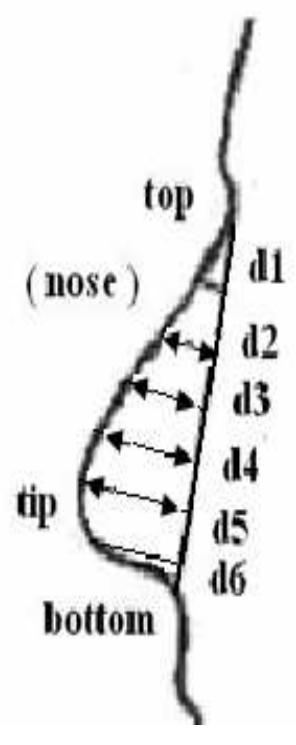

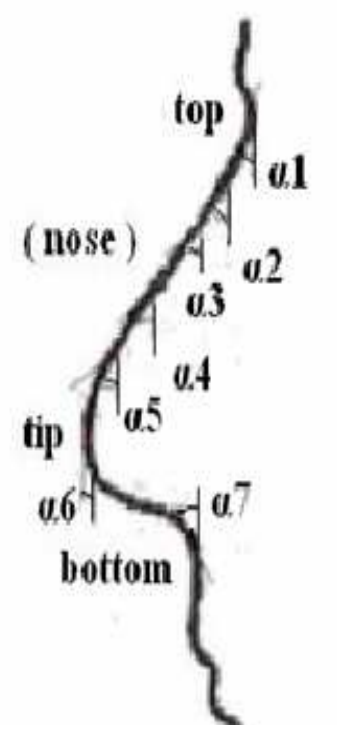

Figure(5): (a) distances of the nose, (b) angles of the nose

\section{EXPERIMENTAL RESULTS AND ANALYSIS}

The face profile database is taken clients from public areas in Cairo city. It contains profile views of 50 persons of RGB image, 3 images for each person are taken with variations of shots, illuminations and different time captured. The size of images was $576 * 768$ pixels.

In this work only 50 images (one image per person) are used in the training dataset, and the remaining 100 images are used as testing datasets. An example of the images collected are shown in figure(6). It is clear that these images are collected under different lighting conditions and with different facial expressions. The database was representing persons with head cover, beard, moustache and different head orientations and different ages from two years old up to sixty. 


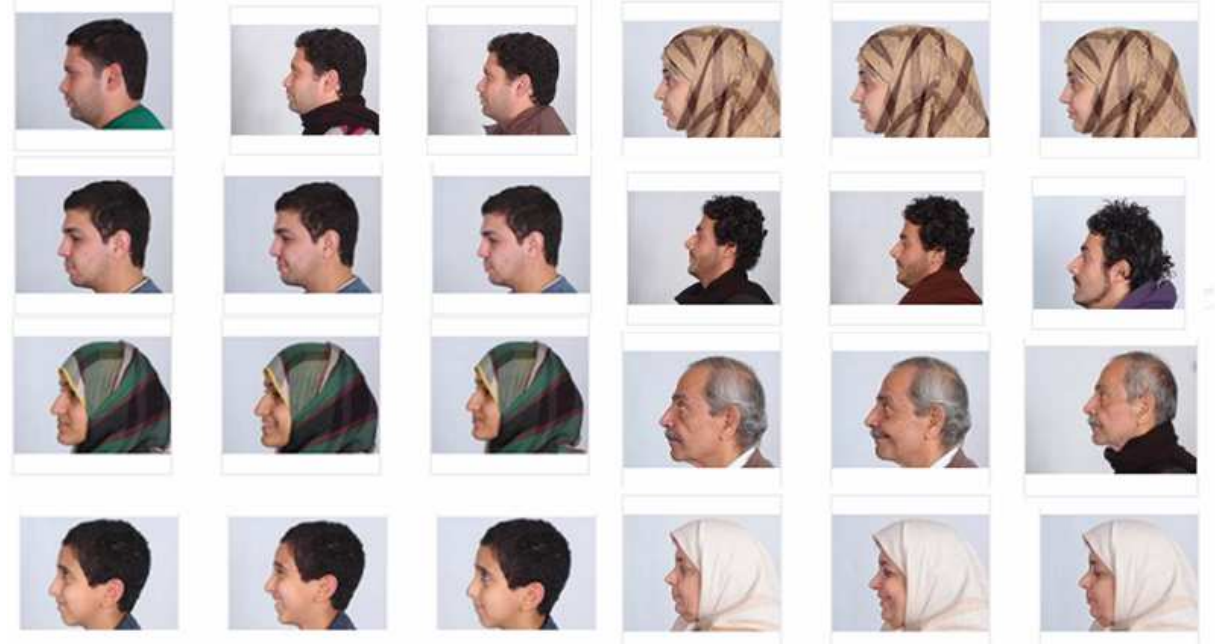

Figure (6) samples from the dataset

The accuracy of the proposed technique is calculated with different values for $\mathrm{m}$ (number of local points). Where the Euclidian distance, equation (2), is measured for $\mathrm{d}$ (distance features) and $\alpha$ (slop features). As shown in Figure(7) the accuracy of the proposed approach reached it's maximum value for $m \geq 8$ with $94 \%$ accuracy for the slop features $\left(\alpha_{1}\right.$ to $\left.\alpha_{m}\right)$ and $96.67 \%$ accuracy for the distance features $\left(d_{1}\right.$ to $\left.d_{m}\right)$.

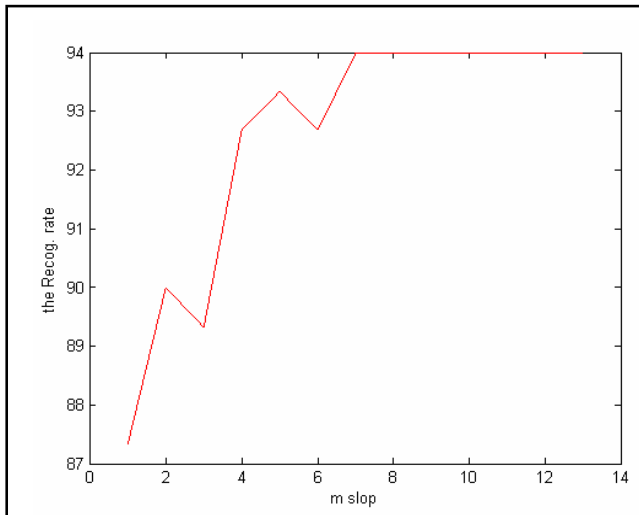

(a)

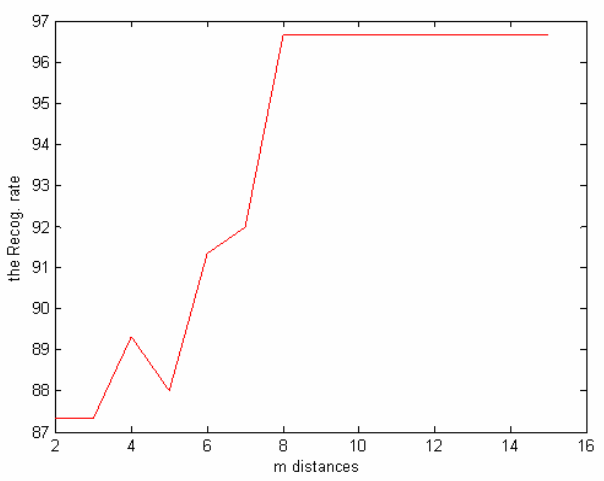

(b)

Figure (7) (a) Accuracy of the angles, (b) Accuracy of the distances

Comparison of studies:

The performance of the proposed technique is compared to another three known facial recognition algorithms for the same and training dataset:

1- Zdravko and Sven [1]: In this approach only four features are selected and used $(\theta 1, \theta 2$, L1 and L2), using simple rule $(\theta 1>0) \&(\theta 2<0) \&(\mathrm{~L} 1>\mathrm{L} 2)$, as shown in figure (8-a). 
2- Maja, Ioannis and Leon [8 ]: In this research ten feature points from the whole profile contour fiducials are used, as in figure (8-b).

3- Mohamed El-Ghotmy [22]: In this research three features $\mathrm{d} 1, \mathrm{~d} 2$ and $\mathrm{d} 3$, as shown in figure $(8-c)$, are selected using the following rule $(\mathrm{d} 1>\mathrm{d} 2>\mathrm{d} 3)$

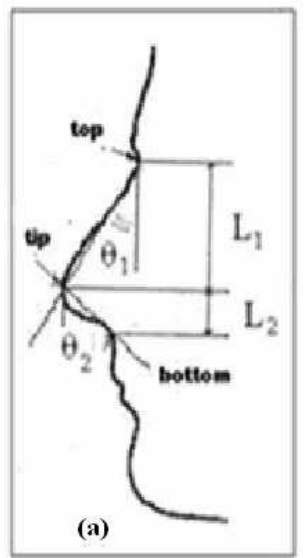

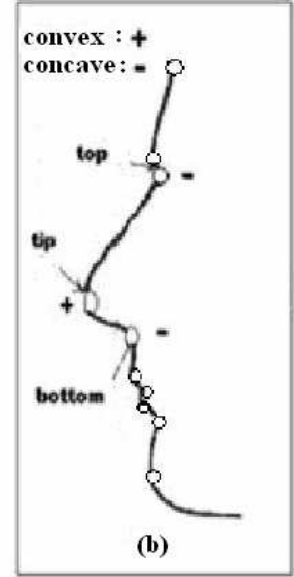

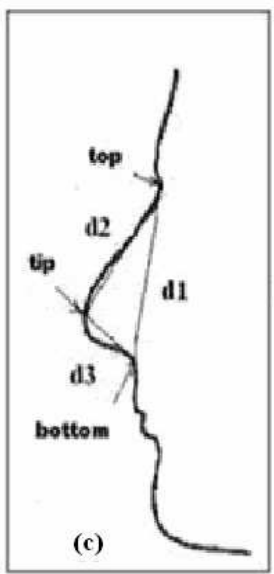

Figure (8) nose features

Table (1) lists the recognition accuracy of each technique, where the performance of the proposed technique is superior to the other techniques by more than 4\%. Also, a computer was used the following configuration: Microsoft Windows XP, Intel ( R) Pentium( R), $1.73 \mathrm{GHz},(795 \mathrm{MHz}, 504 \mathrm{MB}$ of RAM). The time consuming is presented in Table (1).

Table 1. Recognition rates with four different methods

\begin{tabular}{|l|c|c|c|c|}
\hline \multicolumn{1}{|c|}{ Method } & $\begin{array}{c}\text { Curvature- } \\
\text { proposed } \\
\text { technique }\end{array}$ & $\begin{array}{c}\text { Nose tip } \\
\text { detection } \\
\text { [Zdravko] }\end{array}$ & $\begin{array}{c}\text { Convexity- } \\
\text { Concavity } \\
\text { Approach } \\
\text { [Maja] }\end{array}$ & $\begin{array}{c}\text { AFPV system } \\
\text { [EI-Ghotmy] }\end{array}$ \\
\hline $\begin{array}{l}\text { Recognition } \\
\text { rate }(\%)\end{array}$ & 96.67 & 90.67 & 92.22 & 88 \\
\hline Time $(\mathrm{sec})$ & $\begin{aligned} 0.8644+0.31 \\
-0.13\end{aligned}$ & $\begin{array}{r}0.55+0.106 \\
-0.081\end{array}$ & $\begin{array}{ll}0.917+0.349 \\
-0.214\end{array}$ & $0.718+0.548$ \\
\hline
\end{tabular}

\section{CONCLUSIONS}

In this paper, curvature-proposed technique is presented. The curve fitting is used to smooth the profile, then nose angles and nose distances are computed, where the fiducial points from the nose can be reliably extracted, and Euclidean distances method is applied to match the face profile contour on the curve fitting value and through the experiments. The used curvature-proposed technique matching method is found to be promising. As a conclusion about this method, there are 28 fiducial points $\&$ distances 
having maximum recognition rate of about $96.67 \%$. The method is simple, fast and showing promising results.

\section{REFERENCES}

[1] Zdravko Liposcak, and Sven Loncaric, " A Scale Space Approach to Face Recognition from Profiles ", University of Zagreb,Unska 3, Zagreb, Croatia,1999.

[2] Zdravko Loposcak, and Sven Loncaric, " Face Recognition From Profiles Using morphological Opertions ", University of Zagreb, Unska 3, Zagreb, Croatia, 1999.

[3] Xiaoli Zhou, Bir Bhanu, and Ju Han, "Human Recognition at a Distance in Video by Integrating Face Profile and Gait", University of California, Springer Verlag Berlin Heidelberg, 2005.

[4] Bir Bhanu, and Xiaoli Zhou, "Face Recognition From Face Profile Using Dynamic Time Warping ", IEEE computer society, University of California, Riverside, 2004.

[5] Yongsheng Gao, and Maylor K.H. Leung, " Human Face Profile Recognition Using Attributed String", Pattern Recognition 35-353-360, Nanyang Technological University,Singapore, 2002.

[6] Frank Wllhof, Stefan Muller, and Gerhard Rigoll, "Recognition of Face Profiles From The Mugshot Database Using A Hybrid Connectionist /HMM Approach", University Duisburg, Germany, 2000.

[7] Frank Wallhoff, and Gerhard Rigoll, "A Novel Hybrid Face Profile Recognition System Using The Feret And Mugshot Databases", IEEE 0-7803-6725-1/01, 2001.

[8] Maja Pantic, Ioannis Patras, and Leon Rothkrantz, "Facial Gesture Recognition in Face Profile Image Sequences", Delft University of Technology, University of Amsterdam, 2002.

[9] Maja Pantic, and Ioannis, "Dynamics of Facial Expression Recognition of Facial Actions and Their Temporal Segments From Face Profile Image Sequences", IEEE Transactions vol 36. No, 2, April 2006.

[10] L.Akarun,B.Gokberk, A.A.Salah, "3D Face recognition for Biometric Applications", Bogazici University, Bebek, Istanbul, Turkey, 2005.

[11] Rein-Lien Hsu, Mohamed Abdel-Mottaleb, and Anil K. Jain, "Face Detection in Color Images", IEEE Transactions on Pattern analysis and machine intelligence, vol.24, No.5, May 2002.

[12] Yin Zhang, Zhi-Hua Zhou, " Cost-Sensitive Face Recognition ", IEEE Transactions on Pattern analysis and machine intelligence, vol.32, No.10, October 2010.

[13] Xiaona Xu, Zhichun Mu, " Multimodal Recognition Based on Fusion of Ear and Profile Face", IEEE computer society , 0-7695-2929, 2007.

[14] I.A. Kakadiaris, H. Abdelmunim, W. Yang, and T. Theoharis, " Profile-Based Face Recognition" IEEE 978-1-4244-2154-1, 2008.

[15] Mohammed H. Mahoor, A-Nasser Ansari, and Mohamed Abdel- Mottaleb, "Multi-Modal (2D And 3D) Face Modeling And Recognition Using Attributed Relational Graph", IEEE 978-1-4244-1764-3, 2008. 
[16] Ansari A-Nasser, Mahoor Mohammed, and Abdel-Mottaleb Mohamed, " 3D Face Mesh Modeling for 3D Face Recognition", State of the Art in Face Recognition, ISBN-3-902613-42-4,pp.250, 2009.

[17] Mohammed H. Mahoor, Steven Cadavid, and Mohamed Abdel- Mottaleb, " Multi- Modal Ear and Face Modeling and Recognition", IEEE 978-1-4244-56543, 2009.

[18] Mohammed H. Mahoor, Mohamed Abdel-Mottaleb, " Face recognition based on 3D ridge images obtained from range data", Pattern Recognition, 42- PP 445451, 2009.

[19] Chaua C. Queirolo, Luciano Silva, Olga R.P. Bellon, and Mauricio Pamplona Segundo, " 3D Face Recognition Using Simulated Annealing and the Surface Interpenetration Measure", IEEE Transactions on Pattern analysis and machine intelligence, vol.32, No.2, February 2010.

[20] Ira Kemelmacher-Shlizerman, Ronen Basri, " 3D Face Reconstruction from a Single Image Using a Single Reference Face Shape", IEEE Transactions on Pattern analysis and machine intelligence, vol.33, No.2, February 2011.

[21] Kardi Teknomo, "The KNN (nearest neighbor) for Smoothing and Prediction", 2006.

[22] Mohamed El-Ghotmy, "Automatic Face Profile Verification System (AFPVS)", Msc Cairo University, May 2007.

\section{التعرف والتحقق على الاضراد من خلال المنظر الجانبي للوجه}

يقام هذا البحث خواريزما للتعرف على الثخص من خلال المنظر الجانبي للوجه حيث أن هناك بعض التص

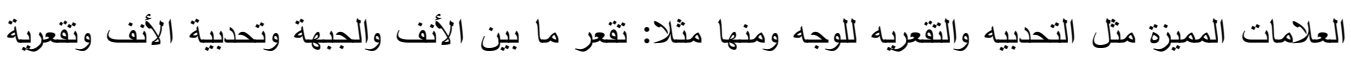

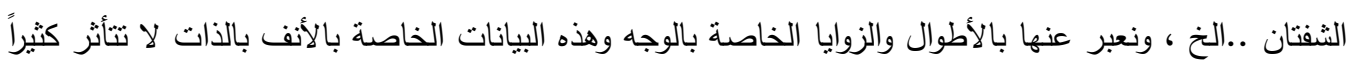
بحالات الوجه الدختلفة مثل الفرح والدهشة وغيرها.

تم استخدم طريقة ال(Nearest Neighbor interpolation) وطريقة ال(Curve Fitting) ـ قاعدة البيانات المستخدمة في هذا البحث تتكون من 50 شخصا ولكل شخص ثلاثة لقطات مختلفة الأوضاع • في هذا البحث كانت نسبة التعرف الصحيحة تساوي 96.67\% ،وبزمن 0.8 من الثانية، وقد قورن بين نتائجه ونتائج ثلاثة أبحاث سابقة مختلفة بثلاثة طرق مختلفة وكانت نتيجته مقبولة ومناسبة بالنسبة لهذه الأبحاث. 